\title{
Wenn-Dann Pläne: eine effektive Planungsstrategie aus der Motivationspsychologie
}

\author{
Tanya Faude-Koivisto \& Peter Gollwitzer
}

\section{$1 \quad$ Einleitung}

Das Setzen von Zielen wird in der wissenschaftlichen Psychologie, in der Praxis des Coachings, sowie in naiven Alltagstheorien als eine gute Strategie betrachtet, eigene Wünsche zu realisieren oder auch Anforderungen gerecht zu werden. Warum misslingt es dann aber immer wieder das Vorhaben einer Gewichtsabnahme oder Ernährungsumstellung in die Tat umzusetzen? Warum hat man schon wieder „Ja“ zu einer neuen Verpflichtung gesagt, obwohl man sich vorgenommen hatte, sich abzugrenzen? Und warum schafft es ihr Coaching Klient zum wiederholten Male nicht, sich die Meinung seiner Teammitglieder anzuhören, obwohl er sich das Ziel gesetzt hat weniger kontrollierend zu sein?

Das Forschungsgebiet der modernen Motivationspsychologie, in deren Zentrum die Erforschung zielbezogener Phänomene steht, kann hierzu hilfreiche Antworten liefern. Die rezente Forschung löst die traditionelle Motivationspsychologie ab, die sich ausschließlich auf die Analyse des Zielsetzens anhand von Erwartungs-Wert-Modellen konzentrierte (Heckhausen, 1989). Dahingegen unterscheidet die moderne Motivationspsychologie zwischen Prozessen des Zielsetzens (,goal setting“") und der Zielrealisierung (,goal striving“") um den jeweils unterschiedlichen zugrunde liegenden psychologischen Prinzipien gerecht zu werden (Gollwitzer, 2006; Gollwitzer \& Moskowitz, 1996; Oettingen \& Gollwitzer, 2001). Eine Unterscheidung auf welche übrigens bereits Kurt Lewin (1926) aufmerksam gemacht hat.

Aus dieser Unterscheidung ergeben sich Antworten der modernen Motivationspsychologie auf die Frage „Wie erreiche ich meine Ziele?““. Zunächst bedarf es für eine erfolgreiche Zielsetzung der intrinsischen Motivationsbildung und der inneren Verpflichtung (,commitment“) einer Person gegenüber dem formulierten Ziel (detaillierte Ausführungen hierzu im Kapitel von Storch in diesem Band). Allerdings weisen zahlreiche Forschungsergebnisse darauf hin, dass eine starke Motivation ein bestimmtes Handlungsergebnis zu erreichen oder ein bestimmtes Verhalten zu zeigen, oft nicht dafür ausreicht, das gesetzte Ziel zu realisieren (Gollwitzer \& Bargh, 1996; Heckhausen, 1989; Kuhl, 1983). Vielmehr ist die Zielrealisierung von Prozessen der Selbstregulation abhängig, da auf dem Weg zwischen dem Setzen und der Verwirklichung eines Ziels unterschiedlichste Herausforderungen dessen Implementierung gemeistert werden müssen (Baumeister, Heatherton \& Tice, 1994; Gollwitzer \& Moskowitz, 1996; Gollwitzer \& Sheeran, 2006; Webb \& Sheeran, 2004). Diese Hürden äußern sich beispielsweise darin, dass man eine günstige Gelegenheit zur Umsetzung seines Ziels nicht erkennt, weil man gerade abgelenkt ist oder seine Aufmerksamkeit auf eine intensive emotionale Erfahrung richtet. Um bei dem vorhergehenden Beispiel des Coaching Klienten zu bleiben, könnte dieser die Gelegenheit verpassen, sich zu Beginn des Meetings zurückzuhalten, da er auf dem Weg ins Besprechungszimmer auf seinem Blackberry eine Mail seines Vermögensverwalters liest, welcher ihm vom weiteren Verfall seiner Aktienpakets berichtet. Desweiteren kann die Realisierung eines Zieles frühzeitig abbrechen, weil andere Projekte im Moment Priorität haben und es einem misslingt, 
das anfängliche Projekt wieder aufzunehmen. So könnte der Coaching Klient sich zwar zu Beginn des Meetings zurücknehmen, wenn an einem bestimmten Punkt „dieses Thema jetzt abschließen um zum Nächsten zu kommen“ für ihn jedoch in den Mittelpunkt rückt, könnte er den Weg zurück zu seinem Vorhaben, nicht alles selbst in die Hand zu nehmen, allerdings verpassen.

Um solche Implementierungsschwierigkeiten gesetzter Ziele zu bewältigen, benötigt es - aus der Sicht der rezenten Motivationspsychologie - den Einsatz von Selbstregulationsstrategien, damit es letztendlich zu einer Zielverwirklichung kommt. Eine sehr effektive Strategie der Selbstregulation stellt das von Gollwitzer $(1993,1999)$ entwickelte Planungsinstrument der Wenn-Dann Pläne dar. Im wissenschaftlichen Kontext bzw. in der experimentellen Forschung werden Wenn-Dann Pläne auch als Vorsätze, Durchführungsintentionen oder ,implementation intentions' bezeichnet. Da sich im angewandten Coaching- und Trainingsbereich der Begriff „Wenn-Dann Pläne“ als geeignet erwiesen hat, wird im Folgenden ausschließlich diese Bezeichnung verwendet.

Gollwitzer (1993, 1999) empfiehlt, die Realisierung von Zielen zu planen, indem gedanklich vorweggenommen wird, wann, wo und auf welche Art und Weise das Ziel erreicht werden soll. Dazu werden gesetzte Zielintentionen („Ich will Z erreichen!“) mit Plänen im Format „Wenn Situation X eintritt, dann will ich das Verhalten Y ausführen!“ ergänzt. Da diese Pläne Verknüpfungen zwischen antizipierten situativen Stimuli und zielgerichtetem Verhalten spezifizieren, wird angenommen, dass die Kontrolle des Handelns vom Selbst weg an die Umwelt delegiert wird. Wenn-Dann Pläne erleichtern die Handlungsinitiierung insofern, als dass es durch diese Verknüpfung zu einer automatischen Auslösung des zielgerichteten Verhaltens kommt sobald die spezifizierte Situation eintritt.

Die Wirkung von Wenn-Dann Plänen als effektive Selbstregulationsstrategie gilt als empirisch abgesichert: zahlreiche Studien aus sehr unterschiedlichen Handlungsfeldern konnten zeigen, dass mit Wenn-Dann Plänen ausgestattete Ziele eine höhere Erfolgsrate aufweisen als Ziele ohne solche Pläne (Übersichten siehe Achtziger \& Gollwitzer, 2006; Gollwitzer \& Sheeran, 2006). Würde die Führungskraft daher ihr Ziel (z.B. „Ich will eine gute Führungskraft sein!“) mit einem Wenn-Dann Plan ergänzen (z.B. „Wenn ich mit meinen Mitarbeitern in einem Meeting sitze und wir mit einem neuen Thema beginnen, dann höre ich mir zunächst die Meinung meiner Mitarbeiter an!“), hätte sie bessere Chancen, das angestrebte Ziel auch tatsächlich zu erreichen.

\section{Das Rubikon-Modell der Handlungsphasen}

Einen entscheidenden Einfluss auf den Forschungsstand der modernen Motivationspsychologie hatte das Rubikon-Modell der Handlungsphasen (Heckhausen \& Gollwitzer, 1987). Die Innovation dieses Modells bestand darin, dass es die beiden Grundthematiken der Motivationspsychologie, nämlich die Wahl von Zielen (,goal setting“) einerseits und Realisierung dieser Ziele (,goal striving“) andererseits, zugleich unterschied und in ein gemeinsames Rahmenmodell integrierte. Das Rubikon-Modell stellt die Grundlage für Gollwitzers (1993, 1996) Konzeptualisierung von Wenn-Dann Plänen dar und wird deshalb hier kurz vorgestellt (für eine detaillierte Ausführung siehe Achtziger \& Gollwitzer, 2006).

Das Rubikon-Modell der Handlungsphasen ist ein motivationspsychologisches Modell zielrealisierenden Handelns und beinhaltet eine umfassende Darstellung des Prozesses der Zielrealisierung. In diesem Modell wird der Handlungsverlauf als ein zeitlicher und somit 
horizontaler Pfad verstanden, der mit den Wünschen einer Person beginnt und mit der Bewertung des jeweils erreichten Zieles endet (Gollwitzer, 1990; Heckhausen \& Gollwitzer, 1987; siehe Abbildung 1).

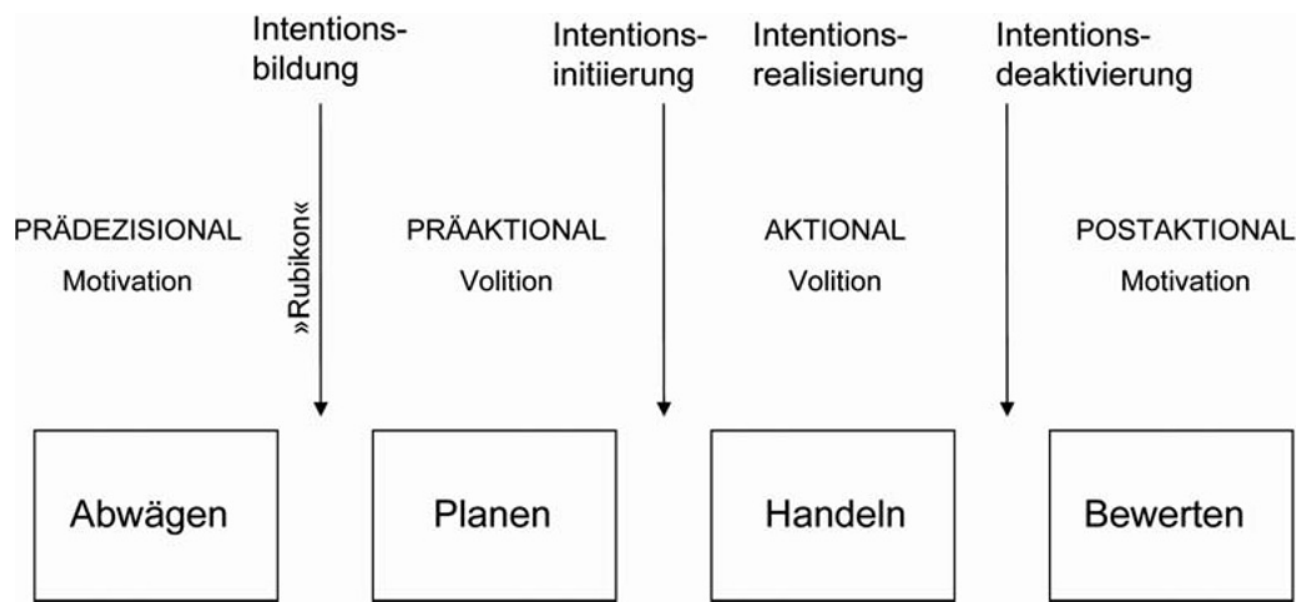

Abbildung 1: $\quad$ Das Rubikon-Modell der Handlungsphasen (Heckhausen \& Gollwitzer, 1987)

Es werden vier aufeinander folgende Handlungsphasen im Rubikon-Modell unterschieden: die prädezisionale, die präaktionale, die aktionale und die postaktionale Phase. Die Phasen unterscheiden sich hinsichtlich der Aufgaben, die sich einem Handelnden jeweils dann stellen, wenn er eine bestimmte Phase erfolgreich abschließen will.

In der prädezisionalen Handlungsphase werden sowohl die Realisierbarkeit bestimmter Wünsche und Anliegen als auch die Wünschbarkeit potenzieller Handlungsergebnisse gegeneinander abgewogen, um dadurch Prioritäten zwischen den verschiedenen Wünschen setzen zu können. Durch diesen Abwägeprozess wird schließlich am Ende der prädezisionalen Phase ein verbindliches Ziel gesetzt, das der Handelnde zu erreichen versucht. Damit wird der „Rubikon“1 vom Wunsch zum Ziel überschritten. Hierbei entsteht ein Gefühl der Verpflichtung, dieses Ziel auch wirklich in die Tat umzusetzen - in der Forschung wird diese Verpflichtung „Commitment“ genannt. An dieser Stelle wird auf das Kapitel von Storch in diesem Band verwiesen. Storch beschreibt ein Zielsetzungsverfahren mit welchem bei der Überquerung des Rubikons die Erzeugung einer starken Zielverpflichtung (und damit einhergehenden intrinsischen Motivation) gewährleistet werden kann.

In der präaktionalen Handlungsphase überlegt sich ein Handelnder, welche Strategien er anwenden soll, um das in der prädezisionalen Phase verbindlich festgelegte Ziel auch wirklich zu realisieren und somit den erwünschten Zielzustand zu erreichen. In dieser Phase werden entsprechend Pläne (z.B. in der Form von Wenn-Dann Plänen) entwickelt, die für das Erreichen des erwünschten Zielzustands förderlich erscheinen. Wenn dieses geplante Verhalten schließlich durch den Handelnden initiiert wird, erfolgt der Übergang in die nächste Phase.

1 Den Begriff des Rubikon wurde in Anlehnung an Julius Caesar gewählt, der 49 v. Chr. mit den Worten "alea jacta est" (lat.: der Würfel ist gefallen), kundtut, dass er nach einer Phase des Abwägens den Entschluss gefasst hat, mit seinen Soldaten den Fluss mit dem Namen "Rubikon" zu überschreiten und damit den Krieg begann. D.h. es gab kein zurück mehr. 
In der aktionalen Handlungsphase besteht die Aufgabe eines Handelnden darin, die geplanten zielfördernden Handlungen auch wirklich durchzuführen und sie zu einem erfolgreichen Ende zu bringen. Dies wird am besten durch ein beharrliches Verfolgen der Zielrealisierung ermöglicht, was eine Anstrengungssteigerung angesichts von Schwierigkeiten impliziert, sowie die konsequente Wiederaufnahme unterbrochener Zielhandlungen erfordert. Ist dies gelungen, schließt sich die vierte Phase an.

In der letzten, postaktionalen Phase des Rubikonmodells bewertet ein Handelnder sein erreichtes Handlungsergebnis, indem er das, was er erreicht hat, mit dem, was er sich ursprünglich gewünscht hat, vergleicht. Ist er damit zufrieden, deaktiviert er das am Ende der prädezisionalen Handlungsphase gesetzte Ziel. Ist er damit nicht zufrieden, senkt er entweder sein Anspruchsniveau und deaktiviert das Ziel oder er behält dieses bei und plant neue Handlungen, die dafür geeignet erscheinen, den erwünschten Zielzustand doch noch zu erreichen. Für eine erfolgreiche Deaktivierung eines nicht erreichten Handlungsziels scheint es förderlich zu sein, wenn der Handelnde ein neues Ziel vor Augen hat, dass das nicht erreichte Ziel ersetzen kann.

Für die Praxis des Coachings bietet sich das Rubikon-Modell als Methode zur Eingangs- und Prozessdiagnostik an. Das Modell erlaubt eine umfassende und wissenschaftlich gut abgesicherte Beschreibung der Entwicklung von Handlungszielen und ermöglicht die Aufgliederung zielgerichteten Verhaltens in eine Abfolge verschiedener, durch spezifische Aufgaben charakterisierte Phasen. Diese Einteilung ermöglicht dem Coach eine Einschätzung seiner Klienten hinsichtlich des aktuellen Standes und dessen, was diese benötigen, um klare Entscheidungen zu treffen und Handlungsfähigkeit zu erlangen. Das RubikonModell wird - in leicht abgewandelter Form - als Instrument zur Eingangs- und Prozessdiagnostik im Coaching bereits im Rahmen der Methodik des Zürcher Ressourcen Modells (Krause \& Storch, 2006) vorgestellt und beschrieben. Daher wird an dieser Stelle nicht näher darauf eingegangen.

\section{Wenn-Dann Pläne: eine Strategie der Selbstregulation für effektives Zielstreben}

Eine Zielsetzung ist entsprechend dem Rubikon-Modell der Handlungsphasen (Heckhausen \& Gollwitzer, 1987) dann erfolgreich verlaufen, wenn sich die Person, welche sich das Ziel setzt, diesem gegenüber innerlich verpflichtet fühlt. Die Aufgabe, die sich einem Handelnden in der ersten (prädezisionalen) Phase stellt, ist entsprechend motivationaler Natur. Häufig führt das geglückte Setzen eines Zieles allein allerdings nicht zur Erreichung des angestrebten Endzustands, da die Aufgabe der präaktionalen Phase des Zielstrebens, die Implementierung des Ziels, misslingt. Manchmal liegt das daran, dass ein Handelnder eine günstige Gelegenheit zur Umsetzung von Zielen in die Realität nicht erkennt, weil er gerade abgelenkt ist und sich seine Aufmerksamkeit z.B. auf intensive emotionale Erfahrung richtet. Zudem wird die Implementierung erschwert, wenn Ziele langfristige Projekte darstellen und deshalb auf lange Zeiträume hinweg immer wieder Anstrengungen erfordern (z.B. der Wechsel in die berufliche Selbständigkeit). Entsprechend dem Rubikon-Modell wird das Problem erfolgreicher Implementierung einer ausgewählten Handlungsweise als von volitionaler Natur betrachtet. Das heißt, es hängt von der Willenskraft einer Person und deren Verfügen über relevante Selbstregulationsstrategienfähigkeiten ab, ob das Verhalten letztendlich implementiert wird. 
Basierend auf diesen Überlegungen schlägt Gollwitzer (1993, 1996) zusätzlich zum Setzen einer Zielintention das Fassen von Wenn-Dann Plänen vor. Sie gelten als ein effektives Instrument zur Förderung der Zielrealisierung, besonders wenn dieser Probleme der Selbstregulation entgegenstehen. Gollwitzer $(1993,1996)$ unterscheidet zwischen Zielintenionen (Absichten, ,goal intentions“) und Wenn-Dann Plänen (Vorsätze, Durchführungsintentionen, ,implementation intentions“). Zielintentionen definieren erwünschte Endzustände (eine erwünschte Leistung oder ein erwünschtes Ergebnis), die bisher noch nicht erreicht worden sind und besitzen das Format „Ich will Z erreichen!“. Somit handelt es sich bei Zielintentionen um „Ziele“ im landläufigen Sinn. Zielintentionen können von der betreffenden Person sowohl abstrakt auf der Haltungsebene (z.B. „Ich will eine gute Führungskraft sein!“) als auch konkret auf der Ergebnisebene (z.B. „Ich will die Meinung meiner Mitarbeiter in meine Entscheidungen mit einfließen lassen!"“) definiert werden (Gollwitzer \& Brandstätter, 1997). Siehe Storchs Kapitel in diesem Band zur Diskussion darüber, wann es sich empfiehlt, Ziele entweder auf der Haltungs- oder der Ergebnisebene zu definieren.

Wenn-Dann Pläne besitzen das Format „Wenn Situation X eintritt, dann will ich das Verhalten Y ausführen!“ und stehen im Dienste von Zielintentionen, d.h. sie sind diesen untergeordnet. Dies impliziert, dass ein Handelnder sich in der Regel zuerst eine Zielintention setzt und sich anschließend einen Wenn-Dann Plan fasst, um durch diesen die Realisierung der Zielintention zu unterstützen. Zielintentionen bilden somit die Grundlage für Wenn-Dann Pläne, oder anders formuliert: Sie sind die Voraussetzung für deren Existenz (Schaal \& Gollwitzer, 2000). Wenn-Dann Pläne sind eine Strategie, in der sowohl das die Realisierung der Zielintention fördernde Verhalten, als auch eine günstige Situation, dieses Verhalten zu zeigen, festgelegt wird. Sie unterstützen die Zielrealisierung, indem sie bestimmen, wann, wo und auf welche Art und Weise das Ziel erreicht werden soll. Die Verknüpfung einer antizipierten Situation mit einem zielfördernden Verhalten im Wenn-Dann Format führt dazu, dass die Kontrolle des Handelns an die spezifizierte Situation delegiert wird.

Um einen Wenn-Dann Plan zu fassen, muss eine Person zunächst eine kritische Situation oder Bedingung antizipieren. Es kann sich hierbei um einen bestimmten Ort, Gegenstand oder Zeitpunkt, eine bestimmte Person, aber auch einen kritischen inneren Zustand wie z.B. sich ärgern handeln. Als nächstes überlegt sich die Person unterschiedliche Möglichkeiten, wie auf den kritischen äußeren oder inneren Stimuli so reagiert werden kann, dass dieses Verhalten zielfördernd ist. Beispielsweise könnte der Coachee mit dem Ziel, seiner Familie mehr Aufmerksamkeit zu schenken, die kritische Situation ,wenn ich auf eine Geschäftsreise gehe und am Ankunftsflughafen in ein Taxi steige“ antizipieren und sich dann ein mögliches zielförderndes Verhalten, wie „dann rufe ich zunächst meine Frau an um ihr mitzuteilen, dass ich gut angekommen bin“"überlegen. Das tatsächliche Fassen des Plans ist dann der mentale Akt der Verknüpfung der antizipierten Situation mit der zielfördernden Handlung in einem „Wenn-Dann“ Format: „Wenn ich auf eine Geschäftsreise gehe und an dem Bestimmungsort ankomme, dann rufe ich zunächst meine Frau an um ihr mitzuteilen, dass ich gut angekommen bin!“.

Zahlreiche Studien aus sehr verschiedenen Handlungsfeldern belegen, dass Personen, die ihre Zielintention mit Wenn-Dann Plänen ergänzen, eine höhere Erfolgsrate bei der Realisierung dieser Zielintentionen aufweisen als Personen, die das nicht tun (Übersichten siehe Achtziger \& Gollwitzer, 2006; Gollwitzer \& Sheeran, 2006). Zudem konnte in einer Meta-Analyse von 94 voneinander unabhängigen Studien zu Wenn-Dann Plänen eine mittlere bis starke Effektstärke (Cohen, 1992) in Bezug auf die Wirkung dieser Pläne gefunden 
werden, und dies über die Wirkung von Zielintentionen alleine hinaus (Gollwitzer \& Sheeran, 2006; Webb \& Sheeran, 2008).

\section{Wie funktionieren Wenn-Dann Pläne?}

Die Wirkung von Wenn-Dann Plänen wird vornehmlich auf zwei psychologische Prozesse zurückgeführt, die sich jeweils auf den „Wenn-Teil“ oder den „Dann-Teil“ des Plans beziehen: die chronische Aktivierung der spezifizierten Situation und die automatische Initiierung des vorgenommenen zielfördernden Verhaltens. Das Fassen eines Wenn-Dann Plans beinhaltet die bewusste Auswahl einer bestimmten zukünftigen Situation oder eines bestimmten Reizes (z.B. eine günstige Gelegenheit, eine schwierige Situation oder einen aversiven inneren Zustand), es wird daher angenommen, dass die mentale Repräsentation dieser Situation hoch aktiviert wird (Gollwitzer, 1993; 1999). Aarts, Dijksterhuis und Midden (1999) wiesen die erhöhte mentale Aktivierung der kritischen Situation mithilfe einer lexikalischen Entscheidungsaufgabe nach. In dieser reagierten Versuchspersonen, die sich einen Wenn-Dann Plan gefasst hatten, schneller auf Wörter, welche in diesem Plan als Situation spezifiziert worden waren als Versuchspersonen, die sich diesbezüglich nur eine Zielintention gesetzt hatten.

Durch die hohe Aktivierung der spezifizierten Situation wird deren kognitive Zugänglichkeit in Folge erhöht. Dies bedeutet, dass die Wahrnehmungs-, Aufmerksamkeits- und Gedächtnisfunktion im Hinblick auf die spezifizierte Situation beeinflusst wird. Sie wird schneller als günstige Gelegenheit zur Durchführung zielfördernden Verhaltens wahrgenommen und zieht selbst bei starker Ablenkung mehr Aufmerksamkeit auf sich. Zusätzlich wird sie besser erinnert, als wenn sie nicht in einem Wenn-Dann Plan spezifiziert worden wäre (z.B. Webb \& Sheeran, 2004). Eine detaillierte Ausführung der ersten empirischen Befunde zu den Folgen der kognitiven Zugänglichkeit der spezifizierten Situation findet sich bei Gollwitzer (1999).

Der zweite Prozess auf den die Wirkung von Wenn-Dann Plänen zurückgeführt wird, ist die Automatisierung der im Plan vorgenommenen Handlung. Gollwitzer (1993) argumentiert, dass das Fassen eines Wenn-Dann Plans (d.h. die Verknüpfung einer kritischen Situation mit einer zielfördernden Verhaltensweise in einem Wenn-Dann Format) ein bewusster Willensakt ist, der die Kontrolle des Verhaltens strategisch vom Handelnden weg an die Umwelt (d.h. die spezifizierte Situation) überträgt. Sie ist somit nicht mehr ,,intern“ von den jeweiligen „inneren Zuständen“ der handelnden Person wie beispielsweise dem Grad an Wachheit oder Energie, sondern „extern“ und somit vom Eintreten der im Plan definierten Situation abhängig. Folglich löst die Situation, sobald diese eintrifft, das Verhalten automatisch aus. Gollwitzer et al. (z.B. Gollwitzer \& Schaal, 1998; Gollwitzer, Fujita \& Oettingen, 2004) sprechen in diesem Zusammenhang auch von ,strategischer“ Automatizität. Die Annahme einer automatischen Realisierung von Wenn-Dann Plänen bedeutet, dass diese bei Eintreffen der in ihnen spezifizierten Situation sofort, effizient und ohne bewusstes Wollen in die Tat umgesetzt werden. Ein Handelnder, der sich einen solchen Plan gefasst hat, muss also nicht erst bewusst und unter Aufwendung kognitiver Ressourcen mühevoll dafür sorgen, dass die geplante zielfördernde Verhaltensweise bei Eintreten der spezifizierten Situation auch wirklich in die Tat umgesetzt wird. Mit anderen Worten, wenn der um die Beziehung zu seiner Frau besorgte Coachee auf einer Geschäftsreise am Flughafen des Ankunftsorts ins Taxi steigt, denkt er sofort und ohne Mühe ,ich rufe jetzt meine Frau an“ 
- ohne nochmals Abwägen zu müssen, ob ein bestimmtes Geschäftstelefonat zuerst erledigt werden muss.

Die Automatisierung der Handlungsinitiierung konnte in unterschiedlichen Studien nachgewiesen werden, in denen das im Wenn-Dann Plan vorgenommene zielfördernde Verhalten unverzüglich (z.B. Gollwitzer \& Brandstätter, 1997; Webb \& Sheeran, 2006), effizient (z.B. Brandstätter, Lengfelder \& Gollwitzer, 2001; Lengfelder \& Gollwitzer, 2001), und ohne bewusstes Wollen (z.B. Bayer, Achtziger, Gollwitzer \& Moskowitz, 2009; Webb \& Sheeran, 2006) ausgelöst wurde, sobald die im Plan spezifizierte Situation eintrat (Übersicht siehe Gollwitzer \& Sheeran, 2006). Beispielswiese konnte die automatische Realisierung von Wenn-Dann Plänen von Lengfelder und Gollwitzer (2001) in einer neuropsychologischen Studie mit Patienten mit Frontallappenschädigung nachgewiesen werden. Diese Patienten haben Probleme mit der bewussten Kontrolle von Handlungen und sind deshalb nur noch in der Lage, habituelle und reflexive, und somit automatisierte Handlungen durchzuführen. In der Studie zeigte sich allerdings, dass bei Patienten, die gebeten wurden sich Wenn-Dann Pläne zu fassen um mit deren Hilfe eine bestimmte Aufgabe schneller zu lösen, die Initiierung des zielfördernden Verhaltens schneller erfolgte als bei gesunden Personen. Daraus kann geschlossen werden, dass Wenn-Dann Pläne automatisch die Kontrolle des Verhaltens induzieren, d.h. es ist hierfür keine bewusste, kognitive Ressourcen verbrauchende Kontrolle notwendig. Besonders interessant sind in diesem Zusammenhang auch Studien, bei denen die im Wenn-Dann Plan spezifizierte Situation subliminal und somit unterhalb der Bewusstseinsschwelle präsentiert wurde. Obwohl hier kein Entschluss zur Durchführung des zielfördernden Verhaltens auf Seiten des Handelnden möglich war, zeigte sich bei der Präsentation dieser Situation eine automatische Handlungsinitiierung (Bayer, Achtziger, Gollwitzer \& Moskowitz, 2009).

Die automatische Handlungsinitiierung bei Wenn-Dann Plänen ist vergleichbar mit der Automatisierung von Verhalten bei Gewohnheitshandlungen („habits“). Beide führen dazu, dass ein mit einer bestimmten Situation oder einem bestimmten Reiz verbundenes Verhalten automatisch ausgeführt wird, sobald diese Situation eintritt bzw. der entsprechende Reiz auftaucht. Man denke hinsichtlich Gewohnheitshandlungen z.B. an das Autofahren: Sobald man sich als geübter Fahrer ins Auto setzt (auslösender Reiz), wird der Zündschlüssel eingesteckt (Verhalten), sobald die Kupplung gedrückt wird (auslösender Reiz), wird der Gang eingelegt (Verhalten), etc. Und all dies geschieht automatisch (d.h. sofort, anstrengungsfrei und ohne bewusst darüber nachdenken zu müssen), so dass sie sich während des Losfahrens auch ungestört mit jemandem unterhalten können. Wenn-Dann Pläne unterscheiden sich von ,habits“ allerdings darin, dass für ihre Etablierung nur ein einziger Willensakt notwendig ist, bei dem ganz bewusst eine enge Verbindung zwischen einer bestimmten Situation bzw. einem bestimmten Reiz und einem zielfördernden Verhalten generiert wird. Bis ein „habit“ sich im offenen Verhalten niederschlägt, sind dagegen viele konsistente Wiederholungen einer bestimmten Verhaltensweise in derselben Situation erforderlich (vgl. Fitts \& Posner, 1967; Newell \& Rosenbloom, 1981). Man spricht daher auch davon, dass das Fassen eines Wenn-Dann Plans eine „sofortige Gewohnheitsbildung“ (,instant habit“) erzeugt (Schaal \& Gollwitzer, 2000).

Dieser Aspekt macht den Einsatz von Wenn-Dann Plänen im Coaching besonders attraktiv - sie erleichtern dem Coachee das Realisieren seines beabsichtigten Verhaltens erheblich und eignen sich vor allem dann, wenn es gilt, alte Automatismen zu umgehen oder neue Automatismen aufzubauen. 


\section{Wenn-Dann Pläne in der Coaching-Praxis}

Die bisherigen Ausführungen implizieren, dass Wenn-Dann Pläne eine effektive Strategie darstellen, um Selbstregulationsschwierigkeiten, denen eine Person nach der Zielsetzung auf dem Weg zum Ziel begegnen kann, erfolgreich zu lösen. Wann und in welcher Form können diese Schwierigkeiten jedoch im Einzelnen auftreten, und wie können Wenn-Dann Pläne helfen? Dies soll im Folgenden erläutert, mit empirischen Daten belegt und mit Coaching Beispielen veranschaulicht werden.

\subsection{Wenn-Dann Pläne fördern die Handlungsinitiierung}

Die erste Selbstregulationshürde, die es nach dem Setzen eines Zieles zu überwinden gilt, besteht in der Initiierung zielfördernder Handlungen. Diese Hürde wird leicht gemeistert, wenn die Zielintention einfach zu realisieren ist, weil z.B. das zu initiierende zielfördernde Verhalten bereits eine Routine darstellt. In solch einem Fall würde der Einsatz von WennDann Plänen keine Zusatzeffekte hinsichtlich des Grades der Zielerreichung zeigen. Allerdings haben Wenn-Dann Pläne eine erhebliche Auswirkung auf den Grad der Handlungsinitiierung, wenn es sich um Zielintentionen handelt, die schwierig zu realisieren sind. Dies ist beispielsweise dann der Fall, wenn neue Verhaltensweisen unter erschwerten Umständen gezeigt bzw. erlernt werden müssen. Starke Wenn-Dann Plan Effekte (d.h. mit Wenn-Dann Plänen ausgestattete Zielintentionen weisen einen höheren Realisierungsgrad auf als Zielintentionen ohne Wenn-Dann Pläne) konnten bisher unter folgenden erschwerten Bedingungen einer Handlungsinitiierung gefunden werden.

Erschwerte situative Faktoren. Zunächst hat es sich gezeigt, dass Wenn-Dann Pläne die Handlungsinitiierung und Handlungsdurchführung auch dann fördern, wenn das zielgerichtete Verhalten zu einem ungünstigen Zeitpunkt gezeigt werden soll. So untersuchten Gollwitzer und Brandstätter (1997) die Realisierung einer Zielintention, die in den Weihnachtsferien von Studierenden in der Tat geschehen sollte. Die Weihnachtsferien stellen für die meisten Studenten einen Zeitraum dar, in welchem mit Arbeit verbundene Aktivitäten meist zugunsten des Zusammenseins mit der Familie und Freunden gemieden werden. Den Versuchspersonen wurde die Aufgabe gestellt, während der Weihnachtsferien einen Bericht über den Heiligabend zu schreiben. Wie erwartet taten das Studierende, die sich einen entsprechenden Wenn-Dann Plan gefasst hatten, signifikant häufiger als Studierende, die sich diesbezüglich nur eine Zielintention gesetzt hatten.

Auf die Coaching-Praxis übertragen könnten Wenn-Dann Pläne z.B. dann hilfreich sein, wenn sich ihr männlicher Coachee das Ziel gesetzt hat, seine körperliche Fitness zu verbessern. Ein Plan könnte dazu lauten „Wenn der Wecker um 6.20 Uhr klingelt, dann stehe ich auf und gehe 30 Min. im Park laufen!“ Die Initiierung seines Handelns sollte durch den Wenn-Dann Plan auch dann abgesichert sein, wenn es beispielsweise draußen regnet. Durch die automatische Handlungsinitiierung wird ein Hin- und Herüberlegen, ob er bei so einem garstigen Wetter laufen gehen soll oder nicht, schlicht umgangen und die Wahrscheinlichkeit, dass er sein Ziel erreicht, in dem er vor der Arbeit Joggen geht, erhöht (natürlich immer vorausgesetzt, dass er sich dem Ziel innerlich verpflichtet fühlt und zur Zielerreichung motiviert ist). 
Erschwerte aufgabenbezogene Faktoren. Desweiteren konnte gezeigt werden, dass Wenn-Dann Pläne die Initiierung von neuem erwünschtem Verhalten auch dann fördern, wenn die Ausführung der Zielhandlung unangenehm ist oder Überwindung erfordert, d.h. die zu lösende Aufgabe bezüglich der Zielerreichung erschwert ist. Beispielsweise stellten Orbell, Hodginks und Sheeran (1997) in einer Studie zur Brustkrebsprävention fest, dass Frauen, die sich die Zielintention gesetzt hatten, innerhalb des nächsten Monats ein Abtasten der Brust durchzuführen, dies zu 53\% taten. Im Gegensatz hierzu zeigten 100\% der Frauen dieses Vorsorgeverhalten, wenn sie sich zusätzlich einen Wenn-Dann Plan gefasst hatten. Dasselbe galt für Studien, welche die Wiederaufnahme von körperlichen Aktivitäten nach Hüftgelenksoperationen (Orbell \& Sheeran, 2000), die Durchführung von sportlichen Übungen (Milne, Orbell \& Sheeran, 2002) und die Aneignung des umweltfreundlichen Verhaltens der Abfallverwertung (Holland, Aarts \& Langendam, 2006) untersuchten. Für das Coaching sind diese Ergebnisse vor allem im Zusammenhang mit umfassenden Veränderungsprozessen innerhalb einer Organisation interessant. Veränderungen von eingefahrenen Verhaltensweisen sind mit großem Unbehagen und Stress verbunden, das bestätigt auch die neurowissenschaftliche Forschung. Große Veränderungen werden vom Gehirn quasi als Bedrohung empfunden (Rock \& Schwartz, 2006). Allerdings ist Erfolg in vielen Geschäftsbereichen in der heutigen Zeit nur dann möglich, wenn das tagtägliche Verhalten der gesamten Belegschaft verändert wird. Würde man in einem solchen Falle die Veränderungsbemühungen damit ergänzen, dass den Mitarbeitern Wenn-Dann Pläne (und optimaler Weise ein Zielsetzungsverfahren, welches die Zielverpflichtung garantiert, siehe Storch in diesem Kapitel) als Instrument zur Selbstregulation näher gebracht werden, wäre der Prozess nicht nur weniger unangenehm und schmerzhaft, sondern auch erfolgreicher.

Erschwertes Erinnern der Handlungsinitiierung. Weiterhin konnten positive Effekte von Wenn-Dann Plänen für zielgerichtete Verhaltensweisen gefunden werden, die leicht vergessen werden, wie z.B. die reguläre Einnahme von Vitamintabletten (Sheeran \& Orbell, 1999) oder das Signieren der einzelnen Arbeitsblätter eines Intelligenztests (Chasten, Park \& Schwarz, 2001). Wenn ein Coachee die Umsetzung einer täglich durchzuführenden Hausaufgabe (beispielsweise eine Aufmerksamkeitsübung) aus dem Coaching demnach mit WennDann Plänen ergänzt, kann die Wahrscheinlichkeit der Umsetzung deutlich erhöht werden.

Personenkreis mit chronischen Schwierigkeiten der Selbstkontrolle. Die Effektivität von Wenn-Dann Plänen lässt sich selbst bei Personen beobachten, die bekannt dafür sind, dass sie aufgrund von Selbstkontrollproblemen Mühe mit der Umsetzung von Zielen in Handeln haben, wie z.B. Opiatabhängige im Entzug und schizophrene Patienten (Brandstätter, Lengfelder \& Gollwitzer, 2001), sowie frontalhirngeschädigte Personen (Lengfelder \& Gollwitzer, 2001). Desweiteren demonstrierten Gawrilow und Gollwitzer (2008) erfolgreich die Wirkung von Wenn-Dann Plänen bei Kindern mit einer Aufmerksamkeitsdefizitstörung (ADHS). Diese Kinder weisen große Defizite bei der Durchführung von Prozessen, die kognitive Ressourcen erfordern, auf. Allerdings zeigt sich, dass Kindern mit ADHS die Kontrolle impulsiven Verhaltens (Stoppreaktion während einer Reaktionszeitaufgabe) mithilfe eines Wenn-Dann Plans genauso gut gelang wie einer Kontrollgruppe von gesunden Kindern.

Aufmerksamkeitsstörungen finden sich allerdings nicht nur bei Kindern. Auch Erwachsene sind davon betroffen, wobei die Störung bei Letzteren meist nicht als solche identifiziert wird. Daher ist die Wahrscheinlichkeit relativ hoch, dass man als Coach irgendwann mit einem Klienten arbeitet, dessen Berufsalltag und Performance durch ADHS enorm erschwert wird. Wenn-Dann Pläne können für solche Personen eine enorme Lebenshilfe darstellen. 


\subsection{Wenn-Dann Pläne unterstützen die Aufrechterhaltung des Zielstrebens}

Bisher haben wir uns damit beschäftigt, wie Wenn-Dann Pläne dazu dienen können, erwünschtes zielförderndes Verhalten auch wirklich in die Tat umzusetzen, um auf diese Weise Zielintentionen zu realisieren. Hierbei lag der Fokus auf der Betrachtung, wie zielförderndes Verhalten überhaupt initiiert werden kann. Allerdings reicht das Initiieren zielfördernden Verhaltens alleine häufig nicht aus um eine Zielintention zu realisieren: Nach der Handlungsinitiierung muss der Prozess des Zielstrebens weiter aufrechterhalten werden. In diesem Sinne muss ein Handelnder das Zielstreben davor schützen, aufgrund attraktiver Ablenkungen wieder beendet zu werden oder konflingierenden schlechten Gewohnheiten zum Opfer zu fallen. Wie Wenn-Dann Pläne das Zielstreben aufrechterhalten, wird nachfolgend geschildert.

Schutz vor äußerer und innerer Ablenkung. Die Forschung auf dem Gebiet des Zielstrebens hat festgestellt, dass der unzureichende Schutz des Zielstrebens vor Ablenkungen eine wichtige Rolle in Bezug auf eine suboptimale Regulation von zielgerichtetem Verhalten spielt. Wenn-Dann Pläne können in diesem Fall Abhilfe schaffen. Die abschirmende Wirkung von Wenn-Dann Plänen vor dem Einfluß äußerer Ablenkungen wurde beispielsweise von Schaal und Gollwitzer (2000) untersucht. Sie konnten zeigen, dass Versuchsteilnehmer, die sich zusätzlich zu einer Zielintention einen Wenn-Dann Plan gefasst hatten, trotz Ablenkung durch gleichzeitig gezeigte Werbefilme mehr Mathematikaufgaben lösen konnten, als Versuchsteilnehmer die sich nur eine Zielintention gesetzt hatten. Dieser Plan Effekt konnte auch zum Schutz vor inneren Ablenkungen, die das Zielstreben behindern, gefunden werden. In einer von Achtziger, Gollwitzer und Sheeran (2008) durchgeführten Studie verhalfen Wenn-Dann Pläne Tennisspielern dazu, störende kognitive, motivationale, physiologische und emotionale Zustände zu regulieren um sich gegenüber einem Gegner besser behaupten zu können.

Wenn-Dann Pläne zur Überwindung von Hindernissen (d.h. zum Schutz vor Ablenkung) werden gebildet, indem das Hindernis im „Wenn-Teil“" des Plans definiert wird und mit einem Verhalten, das sich zur Bewältigung oder zum Umgehen des Hindernisses eignet, im „Wenn-Teil“" verbunden wird.

Kontrolle unerwünschten Verhaltens. Wenn das Streben nach einer Zielintention durch konflingierendes Verhalten (wie z.B. schlechten Gewohnheiten) bedroht wird, sollten WennDann Pläne gefasst werden, die in der Lage sind, ablenkende Reize zu ignorieren. WennDann Pläne haben sich zur Unterdrückung spontaner Aufmerksamkeitsreaktionen (z.B. Gollwitzer \& Schaal, 1998) und bei der Kontrolle stereotyper und vorurteilshafter Reaktionen (Stewart \& Payne, 2008) als geeignet erwiesen. Beispielsweise gelang es Gollwitzer, Sheeran, Michalski und Seifert (im Druck) nachzuweisen, dass Wenn-Dann Pläne in der Lage sind die Anwendung von Stereotypen zu unterbinden. Sie überprüften inwiefern die Benachteiligung weiblicher Bewerber auf Arbeitsplätze in technischen Bereichen durch Wenn-Dann Pläne aufgehoben werden kann. Im Rahmen dieser Studie wurden Informatikstudenten Bewerbungsunterlagen und ein Anforderungsprofil für die Stelle eines Informatikers vorgelegt. Die Hälfte der Bewerbungsunterlagen war mit dem Namen einer Frau, die andere Hälfte mit dem Namen eines Mannes versehen. In einer Vorstudie hierzu wurden den Bewerbungsunterlagen ausschließlich männliche Namen zugeordnet und es zeigte sich, dass sie dann als gleichwertig (im Sinne einer gleichen Eignung auf die ausgeschriebene Stelle) beurteilt wurden. Wurden nach dem Zufallsprinzip jedoch männliche und weibliche Namen auf die Bewerbungsunterlagen verteilt, entschieden sich die Informatikstudenten deutlich häufiger für die Einstellung 
von männlichen Bewerbern und benachteiligten dadurch die weiblichen Bewerber. Nur einer Gruppe von Informatikstudenten, die sich den Plan gefasst hatte „Wenn ich die Eignung eines Bewerbers oder einer Bewerberin beurteilen muss, ignoriere ich deren Geschlecht!“ gelang es, diesen Bias zu überwinden

Grundsätzlich werden Wenn-Dann Pläne zur Kontrolle unerwünschten Verhaltens meist so formuliert, dass zunächst die kritische Situation im „Wenn-Teil“ spezifiziert wird und im „Dann-Teil“" entweder das vom Handeln erwünschte Verhalten, das durch die Ablenkung

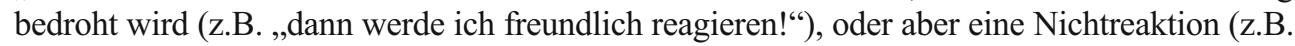
„dann werde ich x ignorieren!“). In der Forschung werden Wenn-Dann Pläne, die auf die Unterdrückung unerwünschter Reaktionen abzielen auch ,Suppressionsvorsätze' genannt. Eine detaillierte Ausführung der Typen von Suppressionsvorsätzen findet sich bei Achtziger und Gollwitzer (2006). Im Coaching können solche Wenn-Dann Pläne beispielsweise dann hilfreich eingesetzt werden, wenn das Ziel des Coachee mit einer gleichberechtigten Behandlung oder Auswahl von Personen im Zusammenhang steht, bzw. stereotypes Verhalten unterdrückt werden soll, oder wenn es darum geht, alte Gewohnheiten mit neuem Verhalten zu ersetzen.

Kontrolle unerwünschter Emotionen. In der, Wenn-Dann Plan'-Forschung konnte weiterhin gezeigt werden, dass auch unerwünschte negative Emotionen kontrolliert werden können. So berichten Schweiger-Gallo, Keil, McCulloch, Rockstroh \& Gollwitzer (2009) von einer Studie zur gesteuerten Kontrolle von Ekel durch Wenn-Dann Pläne. Den weiblichen Versuchspersonen wurden emotionsauslösende Bilder des „International Affective Picture System“ (IAPS; CSEA, 1999) präsentiert. Ein Teil dieser Bilder, nämlich Fotografien von verletzten und verstümmelten Personen, aktivierte die Emotion „Ekel“. Mithilfe eines Wenn-Dann Plans gelang es den Versuchspersonen jedoch ihren Ekel zu unterdrücken, während das mit der Zielintention alleine nicht erreicht werden konnte.

Man stelle sich einen weiblichen Coachee vor, die zwar durch ihr strategisches Denken und Wissen brilliert, durch ihr aggressives Verhalten jedoch nicht in der Lage ist, konstruktive Beziehungen zu ihren Teamkollegen aufzubauen. Würde sie ihr Ziel „Ich will die Beziehung zu meinen Kollegen verbessern!“ durch den Plan „Wenn ich die Wut in mir aufsteigen spüre, dann ignoriere ich diese und sage erstmals nichts!“ ergänzen, hätte sie viel mehr Freiraum, angemessen und sozialförderlich zu reagieren als durch unkontrolliert emotionale Reaktionen ihren Arbeitsplatz zu gefährden.

Blockierung nachteiliger Selbstzustände. In der bisher präsentierten Forschung wurde im „Wenn-Teil“ des jeweiligen Plans eine kritische Situation definiert, die mit einem „Wenn-Teil“ verbunden worden war, der zur Unterbindung unerwünschter Reaktionen diente. Wenn-Dann Pläne können einen Handelnden jedoch auch durch eine völlig andere Vorgehensweise vor unerwünschten Reaktionen schützen. Anstatt die Unterdrückung unerwünschter Reaktionen im „Wenn-Teil“ zu definieren, kann das zielgerichtete Verhalten auch so formuliert werden, dass es darauf ausgerichtet ist, das aktuelle Streben nach Realisierung einer Zielintention zu stabilisieren. Wenn man z.B. müde und erschöpft ist oder an Jet-Lag leidet, kann eine fachliche Diskussion über widersprüchliche Meinungen schnell zu einer hitzigen Diskussion werden, obwohl alle Beteiligten dies nicht wünschen. Wurde jedoch schon im Voraus geplant, wie man mit konträren Meinungen konstruktiv umgehen will (z.B. „Wenn ich in der Besprechung bin, dann gehe ich mit dem Meinungsaustausch sachlich um!“), sollten die Selbstzustände Müdigkeit und Erschöpfung keinen negativen Einfluss auf den Ablauf der Diskussion haben. Diese Annahmen wurden in einer Reihe von Studien überprüft. Mithilfe von Wenn-Dann Plänen wurden negative Effekte von Unvoll- 
ständigkeitserleben hinsichtlich einer erwünschten Identität und negative Effekte einer verminderten Selbstregulationskapazität überwunden (Übersicht siehe Achtziger \& Gollwitzer, 2006). Beispielsweise konnten Webb und Sheeran (2003) die Kompensation von verminderter Selbstregulationskapazität durch das Fassen eines Wenn-Dann Plans demonstrieren. Eine verminderte Selbstregulationskapazität wurde dadurch hergestellt, dass die Versuchspersonen sich auf ihr ,schwächeres“ Bein stellen und gleichzeitig von 1.000 in Siebenerschritten rückwärts zählen sollten. Versuchspersonen in der Kontrollgruppe standen ganz normal auf zwei Beinen und zählten in Fünferschritten vorwärts bis 1.000. Die Zielintention aller Versuchspersonen bestand darin, während einer Stroop-Aufgabe ${ }^{2}$ so schnell wie möglich die Farbe zu benennen, in der dargebotene Wörter geschrieben worden waren. Versuchspersonen in der Planbedingung fassten zusätzlich den Wenn-Dann Plan „Sobald ich ein Wort sehe, dann ignoriere ich dessen Bedeutung und nenne die Farbe, in der es abgebildet ist!“. Bei Versuchspersonen der Planbedingung ließ sich keine verminderte Selbstregulationskapazität beobachten: Sie sprachen die Farbe der Wörter genauso schnell aus wie Versuchspersonen, bei denen die Selbstregulationskapazität nicht vermindert worden war. Versuchspersonen, die sich nur die Zielintention gesetzt hatten, waren gegen den negativen Effekt der verminderten Selbstregulationskapazität nicht geschützt. Sie zeigten deutlich schlechtere Farbnennungsleistungen in der Stroop-Aufgabe.

Blockierung schädlicher Einflüsse der Umgebung. Das Zielstreben einer Person ist nicht nur durch beeinträchtigende Selbstzustände (z.B. Müdigkeit, Unvollkommenheitserleben), sondern auch durch ungünstige situative Bedingungen beeinflusst. Es gibt viele Situationen, die einen negativen Effekt auf die Zielerreichung haben ohne dass die Person, welche das Ziel erstrebt, dies bewusst bemerkt. Man spricht in diesem Falle von „Priming“. Ein Beispiel für Priming ist das Phänomen des „,social loafing“. Darunter wird die Tatsache verstanden, dass sich Personen weniger bei der Arbeit anstrengen wenn die Verantwortung für das Ergebnis nicht auf die einzelnen beteiligten Person zurückgeführt werden kann. Da Personen sich dieses Phänomens in der Regel nicht bewusst sind, sind sie auch nicht in der Lage, Pläne zu fassen, welche die „social loafing“ Situation als „Wenn-Teil“ spezifiziert. Demnach sind Wenn-Dann Pläne die auf das Unterdrücken eines ,social loafing“ Verhaltens abzielen, nicht brauchbar. Die Alternative dazu ist, dass Personen Wenn-Dann Pläne fassen, die festlegen wie die vorgesehene Aufgabe ausgeführt werden soll, um dadurch negative situative Einflüsse effektiv zu hemmen.

Die Annahme, dass Zielstreben, welches durch Wenn-Dann Pläne ausformuliert bzw. geplant wird, vor situativen Einflüssen geschützt ist, konnte beispielsweise in Bezug auf die Überwindung von Verlustframingeffekten gezeigt werden. Personen können sich die erwünschten Ergebnisse ihrer Handlungen als Gewinne oder Verluste vorstellen (Kahneman \& Tversky, 1979). Forschungsergebnisse auf dem Gebiet der Konfliktlösung haben gezeigt, dass kognitive Prozesse, die aufgrund eines so genannten „Verlustframings“ oder eines „Gewinnframings“ entstehen, einen starken Einfluss auf Verhandlungsergebnisse haben (De Dreu, Carnevale, Emans \& van de Vliert, 1994). Verlustframing führt häufig zu vergleichsweise unfairen Vertragsabschlüssen und anderen negativen Effekten. Trötschel \& Gollwitzer (2007) konnten nachweisen, dass die negativen Effekte des Verlustframings

2 In einer Stroop-Aufgabe werden Worte von Farben (z.B. rot, blau, grün) in unterschiedlichen Farben dargestellt. Wenn ein Wort in einer zu ihrer semantischen Bedeutung unterschiedlichen Farbe dargestellt wird (z.B. das Wort "rot" erscheint in blauer Farbe) brauchen Versuchspersonen in der Regel länger die Farbe zu nennen als wenn das Wort in der kongruenten Farbe erscheint (Stroop, 1935) 
durch prosoziale Ziele, wie z.B. das Ziel, eine faire oder integrative Lösung zu finden, erfolgreich überwunden werden können, wenn diese durch entsprechende Wenn-Dann Pläne unterstützt werden.

\subsection{Das Formulieren von Wenn-Dann Plänen}

Hinsichtlich des Differenziertheitsgrades der Formulierung von Wenn-Dann Plänen gilt, dass die zu identifizierende Situation im „Dann-Teil“ des Planes möglichst spezifisch ausformuliert werden sollte, da mit höherem Konkretheitsgrad eine höhere Aktivierung der Situation zu erwarten ist (Gollwitzer, Wieber, Myers \& McCrea, in Vorbereitung). Allerdings muss die Spezifizierung im Hinblick auf die Häufigkeit des Auftretens abgewogen werden, da spezifische Situationen teilweise weniger häufig erscheinen als weniger spezifische. Desweiteren ist darauf zu achten, dass die gewählte Situation in keiner Art und Weise ambivalent, sondern für den Handelnden eindeutig als solche identifizierbar ist.

In Bezug auf den „Wenn-Teil“ wird angenommen, dass das Abstraktionsniveau keinen Einfluss auf die Wenn-Dann Plan Wirkung hat. Die Spezifizierung von einfachem Verhalten (z.B. das Drücken einer Taste) sowie von komplexem Verhalten (z.B. die Ausführung einer Verhaltenssequenz) im „Dann-Teil“ der Pläne erwies sich als effektiv für die Förderung der Zielerreichung (Gollwitzer et al., in Vorbereitung). Allerdings ist darauf zu achten, dass das Verhalten, welches im „Dann-Teil“ eines Plans spezifiziert wird, innerhalb der eigenen Kontrolle der Person liegt und für das Erreichen der jeweiligen Absicht auch geeignet ist.

Ein weiterer Punkt auf den geachtet werden sollte, ist die mögliche Interferenz seitens der spezifizierten Situation und des zielfördernden Verhaltens. Grundsätzlich gilt, dass entsprechend der Zahl der aktivierten Elemente die entsprechende Zahl anderer Elemente gehemmt wird (vgl. Kruglanski et al., 2002). Daher empfiehlt es sich beispielsweise nicht, mehrere Handlungsweisen mit der gleichen Situation zu verknüpfen (außer die Handlungen haben eine logische Abfolge).

Es wird häufig gefragt, wie die Wenn-Dann Pläne technisch zu fassen seien. Dazu lässt sich zunächst sagen, dass das „Wenn-Dann“ Format der Pläne unbedingt einzuhalten ist. Es reicht also nicht, lediglich zu spezifizieren wann, wo und wie man ein Ziel zu erreichen beabsichtigt (Gollwitzer et al., in Vorbereitung). Desweiteren kann ein Plan verankert werden in dem man ihn entweder aufschreibt, laut ausspricht oder drei Mal mental wiederholt. In WennDann Plan Studien wird das Fassen der Pläne meist so gehandhabt, dass Versuchspersonen bei vorgegebenen Plänen diesen zunächst auf einer Seite lesen, und im Anschluss darum gebeten werden, auf einer nächsten Seite den Satz „Wenn , dann ___ ‘ entsprechend des vorher gelesenen Plans schriftlich zu ergänzen. Wird der Inhalt der Pläne von den Versuchspersonen selbst spezifiziert, wird zunächst eine günstige Situation und ein zielgerichtetes Verhalten definiert und aufgeschrieben und in einem nächsten Schritt in einem Wenn-Dann Format nochmals schriftlich festgehalten. Teilweise werden die Versuchspersonen auch darum gebeten, den Plan entweder nur oder zusätzlich zum Aufschreiben laut auszusprechen oder drei Mal innerlich leise vor sich herzusagen. Das Aufschreiben und das (laute oder innerliche) Sagen der Pläne dienen einerseits der Überprüfung, ob die Versuchspersonen den Plan erinnern, sowie der besseren kognitiven Verankerung. Wenn-Dann Plan Effekte wurden für die schriftliche und mentale Form der Verankerung gefunden (siehe hierzu auch im nächsten 
Abschnitt unter Moderatoren der Plan Wirkung). Für das Coaching wird empfohlen, die Form der Verankerung von Wenn-Dann Plänen individuell auf den Coachee abzustimmen, je nachdem auf welche Art und Weise diese(r) besser lernt. Zusätzlich lässt sich noch ergänzen, dass es hinsichtlich der Wirkung von Wenn-Dann Plänen keinen Unterschied macht, ob diese vorgegeben oder von der Person selbst zusammengesetzt werden (immer vorausgesetzt, dass das Ziel-Commitment vorhanden ist). Das heisst ein Coach kann gegebenenfalls auch dem Coachee Pläne vorgeben.

\section{Moderatoren der Wirkung von Wenn-Dann Plänen}

Die Stärke der Wenn-Dann Plan Wirkung hängt von verschiedenen Moderatoren ab. So wurde anhand von mehreren Studien gezeigt, dass Wenn-Dann Plan Effekte von dem Commitment gegenüber der dem Plan übergeordneten Zielintention und der kognitiven Aktivierung der Zielintention abhängen. Orbell und Mitarbeiter (1997) berichten in diesem Zusammenhang, dass die positiven Effekte eines Wenn-Dann Plans auf die Durchführung von Maßnahmen zur Brustkrebsfrüherkennung sich nur bei den Frauen zeigten, welche im Sinne einer Zielintention stark beabsichtigten, eine Selbstuntersuchung der Brust durchzuführen. Desweiteren entdeckten Sheeran und Mitarbeiter (2005), dass diese Effekte nur dann auftreten, wenn sich die im Wenn-Dann Plan zugrunde liegende Zielintention in einem aktivierten Zustand befindet. Der Plan, in einem Intelligenztest nach der Bearbeitung eines Items sofort und ohne Pause zum nächsten zu wechseln, hatte nur dann einen Effekt auf die Geschwindigkeit der Aufgabenbearbeitung, wenn das Ziel, möglichst schnell zu arbeiten, aktiviert, das heißt gesetzt wurde.

Ein weiterer Moderator für die Wirkung ist das Commitment auf den jeweils gefassten Wenn-Dann Plan. Das heißt, ein Wenn-Dann Plan hat nur dann eine zielführende Wirkung, wenn sich eine Person dem Ziel und dem dazu gefassten Plan innerlich verpflichtet fühlt. Zusätzlich ließ sich beobachten, dass Wenn-Dann Plan Effekte umso offensichtlicher sind, je schwieriger es ist, ein zielförderndes Verhalten zu initiieren (z.B. Gollwitzer \& Brandstätter, 1997). Auch die Stärke der Verknüpfung zwischen dem „Wenn-Teil“ eines Plans und seinem „Dann-Teil“ dürfte die Wirkung dieser Pläne positiv beeinflussen. Wenn ein Handelnder viel Zeit und Konzentration dafür aufwendet, den Wenn-Dann Plan im Langzeitgedächtnis zu enkodieren oder wenn er ihn innerlich immer wiederholt, sollte sich eine stärkere Verbindung zwischen den beiden Planteilen ausbilden, die wiederum stärkere Plan Effekte generieren sollten als eine schwache Verbindung zwischen beiden Teilen. Experimentelle Studien zu dieser Fragestellung liegen jedoch noch nicht vor.

Abgesehen von den genannten Moderatoren in Bezug auf die Merkmale der Wenn-Dann Pläne und der ihnen übergeordneten Ziele, scheint die Wirkung dieser Pläne auch von Selbstwirksamkeitsüberzeugungen und Persönlichkeitsmerkmalen abzuhängen. Zunächst hat sich gezeigt, dass eine schwache Selbstwirksamkeitsüberzeugung bezüglich der Realisierung des entsprechenden Ziels die Wirkung von Wenn-Dann Plänen einschränkt (Wieber, Odenthal \& Gollwitzer, im Druck). Desweiteren scheinen Personen, die ihr Verhalten entsprechend dem Maßstab anderer Personen bewerten, nicht von Wenn-Dann Plänen zu profitieren (Powers, Koestner \& Topciu, 2005). Abschließend lässt sich ergänzen, dass das Persönlichkeitsmerkmal der Gewissenhaftigkeit („,consciousness“) die Wirkung von Wenn-Dann Plänen beeinflusst. Während Personen mit einem niedrigen Grad an Gewissenhaftigkeit enorm von 
Wenn-Dann Plänen profitieren, trifft dies für Personen, die sehr gewissenhaft sind, nicht zu. Es wird angenommen, dass die überdurchschnittliche Leistung von Personen mit einer hohen Gewissenhaftigkeit wenig Spielraum für eine Leistungsoptimierung beinhaltet, man spricht hier auch von einem Deckeneffekt (Webb, Christian \& Armitage, 2007).

\section{Potentielle Kosten von Wenn-Dann Plänen}

Wie bisher dargestellt wurde, erleichtert das Fassen von Wenn-Dann Plänen die Zielrealisierung auf vielfältige Art und Weise. Aber welche Kosten sind damit verbunden? In diesem Zusammenhang steht die Möglichkeit einer Verhaltensrigidität im Vordergrund, die sich bei Flexibilität erfordernder Aufgaben ungünstig auswirken könnte (Gollwitzer, ParksStamm, Jaudas \& Sheeran, 2008).

Zunächst stellt sich die Frage, ob eine Person, die sich einen Wenn-Dann Plan gefasst hat, in der Lage ist, alternative geeignete Gelegenheiten zu nutzen, die nicht im Wenn-Dann Plan spezifiziert wurden. Dass dies der Fall ist, konnte in einer Reihe von Studien bestätigt werden (Achtziger \& Gollwitzer, 2006). Das Fassen eines Wenn-Dann Plans resultiert nicht darin, dass sich Versuchspersonen von einem sich verändernden Kontext oder von einer unerwartet auftauchenden günstigen Situation zur Zielrealisierung nicht beeinflussen lassen. Vielmehr kann angemessen auf die neue Situation reagiert werden, anstatt rigide an der vorgenommenen Gelegenheit festzuhalten. Das heißt, dass sich z.B. eine Person mit dem Plan „Wenn mein Chef zu unserem Termin in mein Büro tritt, dann werde ich mein Austreten aus der Firma ansprechen!“, auch dann das spezifizierte Verhalten zeigt, wenn sich die Situation ändert und der Chef ihm über E-Mail mitteilt, dass sie sich nicht in seinem Büro, sondern in der Kantine treffen.

Da Wenn-Dann Pläne zu einer Automatisierung der Handlungsinitiierung führen, welche kein bewusstes Wollen mehr benötigt, stellt sich des Weiteren die Frage, inwiefern das in einem Wenn-Dann Plan spezifizierte Verhalten abhängig von der Stärke und der Aktivierung des zugehörigen Zieles ausgelöst wird. Das heißt, wird das im Plan spezifizierte zielfördernde Verhalten auch dann noch ausgelöst, wenn das Ziel bereits erreicht wurde oder nur noch schwach ausgeprägt ist? Wie bereits an anderer Stelle erwähnt, ist auch diese Form der Verhaltensrigidität nicht der Fall. Zum Beispiel fanden Gollwitzer et al. (2002), dass positive Effekte eines Wenn-Dann Plans auf das Wiedererinnern der spezifizierten Situation nur dann auftraten, wenn das dem Plan übergeordnete Ziel noch in die Tat umgesetzt werden musste. War es bereits anderweitig realisiert worden, zeigte sich kein WennDann Plan Effekt auf die Gedächtnisleistung. Zusammengefasst lässt sich daher sagen, dass Wenn-Dann Pläne nicht zu rigidem Handeln führen, sondern sie passen sich flexibel an sich bietende alternative Gelegenheiten, sowie die Stärke und die Aktivierung des übergeordneten Ziels an.

\section{Ausblick}

Das Fassen von Wenn-Dann Plänen (Gollwitzer, 1993, 1996) stellt eine effektive Planungsstrategie dar, die es Personen erleichtert, Selbstregulationsschwierigkeiten auf dem Wege der Zielrealisierung erfolgreich zu lösen. Die Forschung auf dem Gebiet der Motivationspsychologie zeigt, dass der Einsatz dieser Planungsstrategie (d.h. die Verknüpfung von 
antizipierten Situationen und zielgerichtetem Verhalten in einem Wenn-Dann Format) Personen dabei hilft, zwei zentrale Selbstregulationshürden zu überwinden: zielgerichtetes Handeln a) zu initiieren und b) aufrechtzuerhalten bzw. von Ablenkungen oder alten Gewohnheiten abzuschirmen. Daher weisen Personen, die ihre Ziele mit Wenn-Dann Plänen ergänzen eine höhere Erfolgsrate bei der Realisierung dieser Ziele auf als Personen, die das nicht tun.

Eine wichtige Voraussetzung dafür, dass Wenn-Dann Pläne einen positiven Effekt auf die Zielerreichung haben, ist das Vorhandensein einer starken Zielintention. Dies bedeutet, dass sich die Person, die sich ein Ziel setzt, sich diesem innerlich verpflichtet fühlt und zu dessen Realisierung intrinsisch motiviert ist. In Bezug auf die Frage „Wie erreiche ich meine Ziele?" lässt sich seitens der Motivationspsychologie daher folgendes festhalten. Zuerst bedarf es einer erfolgreichen Zielsetzung, d.h. der Formulierung eines Ziels welches Commitment und intrinsische Motivation beinhaltet. Hierfür eignet sich das Bauen von Haltungszielen, bzw. so genannten Motto-Zielen (siehe Storch in diesem Band). Zweitens gilt es nach der Zielsetzung Selbstregulationsherausforderungen auf dem Weg zum Ziel erfolgreich zu meistern. Eine theoretisch und empirisch gut abgesicherte Strategie zur Lösung dieser zweiten, selbstregulatorischen Aufgabe stellen die von Gollwitzer $(1993,1996)$ entwickelten Wenn-Dann Pläne dar.

Ein wichtiges Thema, das noch weiterer Forschung bedarf, bezieht sich auf die Wirkungsdauer von Wenn-Dann Plänen. Man spricht im Zusammenhang von Wenn-Dann Plänen auch von einer ad hoc Gewohnheitsbildung, da es durch nur einen bewussten Willensakt zur Etablierung einer automatischen Handlungsinitiierung kommt. Es stellt sich daher die Frage, wie lange dieser Effekt anhält. Stadler, Oettingen und Gollwitzer (2009) fanden hierzu in einer Studie zur körperlichen Aktivität bei Frauen ein viel versprechendes Ergebnis. Versuchspersonen, welche sich zu ihrem Ziel, mehr Sport zu treiben, einen Wenn-Dann Plan gefasst hatten, waren bereits nach 1 Woche körperlich aktiver, wobei dieser Effekt über den Verlauf von weiteren 4 Monaten verzeichnet werden konnte. Es kann daher davon ausgegangen werden, dass die Wirkung von Wenn-Dann Plänen über längere Zeiträume anhält, es benötigt hierzu allerdings noch mehr Langzeitstudien.

Zukünftige Forschung sollte zudem versuchen weitere effektive Selbstregulationsstrategien für die Lösung von Problemen der Zielimplementierung zu identifizieren und Unterschiede der Wirkungsweise zu Wenn-Dann Plänen untersuchen. Im Ansatz ist dies bereits geschehen. Faude-Koivisto, Wuerz und Gollwitzer (2008) stellten die Selbstregulationsstrategie der mentalen Simulation der des Wenn-Dann Planens gegenüber und stellten fest, dass beide jeweils unterschiedliche Bewusstseinslagen auslösen (eine abwägende versus eine planende). Mögliche Implikationen dafür, wann und für welches Selbstregulationsproblem sich die Anwendung welcher Strategie empfiehlt, wurden andiskutiert. Es bedarf zur konkreten Differenzierung jedoch weiterer Studien.

Der Einsatz von Wenn-Dann Plänen im Coaching-Prozess ermöglicht es einem Coach, Coaching Klienten in ihrer Selbstregulationsfähigkeit zu unterstützen, in dem die Klienten lernen, ihr Zielstreben durch Wenn-Dann Pläne auszuformulieren. Wenn-Dann Pläne verhelfen den Coachees letztendlich dazu, ihre persönlichen und beruflichen Ziele mit einer sehr einfachen aber enorm effektiven Strategie müheloser zu erreichen. 


\section{Literatur}

Aarts, H., Dijksterhuis, A. P., \& Midden, C. (1999). To plan or not to plan? Goal achievement of interrupting the performance of mundane behaviors. European Journal of Social Psychology, 29, 971-979.

Achtziger, A. \& Gollwitzer, P. M. (2006). Motivation und Volition im Handlungsverlauf . In J. Heckhausen \& H. Heckhausen (Eds.), Motivation und Handeln (pp. 277-302). Berlin: Springer Verlag.

Achtziger, A., Gollwitzer, P. M., \& Sheeran, P. (2008). Implementation intentions and shielding goal striving from unwanted thoughts and feelings. Personality and Social Psychology Bulletin, 34, 381-393.

Baumeister, R.F., Heatherton, T.F. \& Tice, D.M. (1994). Losing control: How and why people fail at self-regulation. San Diego: Academic Press.

Brandstätter, V., Lengfelder, A. \& Gollwitzer, P. M. (2001). Implementation intentions and efficient action initiation. Journal of Personality and Social Psychology, 81, 946-960.

Bayer, U. C., Achtziger, A., Gollwitzer, P. M. \& Moskowitz, G. (2009). Responding to subliminal cues: Do if-then plans cause action preparation and initiation without conscious intent? Social Cognition, 27, 183-201.

Chasteen, A. L., Park, D., \& Schwarz, N. (2001). Implementation intentions and facilitation of prospective memory. Psychological Science, 12, 457-461.

Cohen, J. (1992). A power primer. Psychological Bulletin, 112, 155-159.

CSEA, (1999). International affective picture system (IAPS): technical manual and affective ratings. Gainesville, Florida: Center for the study of emotion and attention, University of Florida.

De Dreu, C. K. W., Carnevale, P. J. D., Emans, B. J. M. \& van de Vliert, E. (1994). Effects of gainloss frames in negotiation: Loss aversion, mismatching, and frame adoption. Organizational behavior and human decision processes, 60, 90-107.

Fitts, P. M. \& Posner, M. I. (1967). Human performance. Oxford, England: Brooks/Cole.

Gawrilow, C. \& Gollwitzer, P. M. (2008). Implementation intentions facilitate response inhibition in ADHD children. Cognitive Therapy and Research, 32, 261-280.

Gollwitzer, P. M. (1990). Action phases and mind-sets. In E. T. Higgins \& R. M. Sorrentino (Eds.), Handbook of motivation and cognition: Foundations of social behavior, 2, (pp. 53-92). New York: Guilford Press.

Gollwitzer, P. M. (1993). Goal achievement: the role of intentions. European Review of Social Psychology, 4, 141-185.

Gollwitzer, P. M. (1996). The volitional benefits of planning. In P. M. Gollwitzer \& J. A. Bargh (Eds.), The psychology of action: Linking cognition and motivation to behavior (pp. $287-312$ ). New York: Guilford.

Gollwitzer, P. M. (1999). Implementation intentions: Strong effects of simple plans. American Psychologist, 54, 493-503.

Gollwitzer, P. M. (2006). Successful goal pursuit. In Q. Jing, H. Zhang, \& K. Zhang (Eds.), Psychological science around the world (Vol. 1, pp. 143-159). Philadelphia: Psychology Press.

Gollwitzer, P. M. \& Bargh, J. A. (Eds.) (1996). The psychology of action: Linking cognition and motivation to behavior. New York: Guilford Press.

Gollwitzer, P. M. \& Brandstätter, V. (1997). Implementation intentions and effective goal pursuit. Journal of Personality and Social Psychology, 73, 186-199

Gollwitzer, P. M. \& Moskowitz, G. B. (1996). Goal effects on action and cognition. In E.T. Higgins \& A. W. Kruglanski (Eds.), Social psychology: Handbook of basic principles. (pp. 361-399) New York: Guilford Press.

Gollwitzer, P. M. \& Schaal, B. (1998). Metacognition in action: The importance of implementation intentions. Personality and Social Psychology Review, 2, 124-136.

Gollwitzer, P. M. \& Sheeran, P. (2006). Implementation intentions and goal achievement: A metaanalysis of effects and processes. Advances in Experimental Social Psychology, 38, 69-119. 
Gollwitzer, P. M., Bayer, U. \& Wicklund, R. A. (2002). Das handelnde Selbst: Symbolische Selbstergänzung als zielgerichtete Selbstverwirklichung. In D. Frey (Ed.), Theorien der Sozialpsychologie (pp. 191-212). Bern: Huber.

Gollwitzer, P. M., Fujita, K., \& Oettingen, G. (2004). Planning and the implementation of goals. In R. Baumeister \& K. Vohs, Handbook of self-regulation (pp. 211-228). New York: Guilford Press.

Gollwitzer, P. M., Parks-Stamm, E. J., Jaudas, A. \& Sheeran, P. (2008). Flexible tenacity in goal pursuit. In J. Shah \& W. Gardner (Eds.), Handbook of motivation science (pp. 325-341). New York: Guilford Press.

Gollwitzer, P. M., Sheeran, P., Michalski, V. \& Seifert, A. E. (im Druck). When intentions go public: Does social reality widen the intention-behavior gap? Psychological Science.

Gollwitzer, P. M., Wieber, F., Myers, A. L. \& McCrea, S. (in Vorbereitung). Maximizing implemention intention effects. In C. R. Agnew, D. E. Carlston, W. G. Graziano, J. R. Kelly (Eds), Then a miracle occurs: Focusing on behavior in social psychology theory and research. New York: Oxford Press.

Heckhausen, H. (1989). Motivation und Handeln. Berlin: Springer.

Heckhausen, H., \& Gollwitzer, P.M. (1987). Thought contents and cognitive functioning in motivational versus volitional states of mind. Motivation and Emotion, 11, 101-120.

Holland, R. W., Aarts, H. \& Langendam, D. (2006). Breaking and creating habits on the working floor: A field-experiment on the power of implementation intentions. Journal of Experimental Social Psychology, 42, 776-783.

Kahneman, D., \& Tversky, A. (1979). On the interpretation of intuitive probability: A reply to Jonathan Cohen. Cognition, 7, 409-411.

Krause, F. \& Storch, M. (2006). Ressourcenorientiert coachen mit dem Zürcher Ressourcen Modell ZRM. Psychologie in Österreich, 26, 32-43.

Kruglanksi, A. W., Shah, J. Y., Fishbach, A., Friedman, R., Chun, W. Y. \& Sleeth-Keppler, D. (2002). A theory of goal systems. In M. P. Zanna (Ed.), Advances in experimental social psychology (Vol. 34, pp. 331-378). San Diego, CA: Academic Press.

Kuhl, J. (1983). Motivation, Konflikt und Handlungskontrolle. Heidelberg: Springer-Verlag.

Lengfelder, A. \& Gollwitzer, P. M. (2001). Reflective and reflexive action control in patients with frontal brain lesions. Neuropsychology, 15, 80-100.

Lewin, K. (1926). Vorsatz, Wille und Bedürfnis. Psychologische Forschung, 7, 330-385.

Milne, S., Orbell, S. \& Sheeran, P. (2002). Combining motivational and volitional interventions to promote exercise participation: Protection motivation theory and implementation intentions. British Journal of Health Psychology, 7, 163-184.

Newell, A. \& Rosenbloom, P. S. (1981). Mechanisms of skill acquisition and the law of practice. In J. R. Anderson (Ed.), Cognitive skills and their acquisition (pp. 1-55). Hillsdale, NJ: Erlbaum.

Oettingen, G. \& Gollwitzer, P. M. (2001). Goal setting and goal striving (pp.329-347). In A. Tesser \& N. Schwarz (Eds.), The Blackwell Handbook of Social Psychology. Oxford: Blackwell.

Orbell, S. \& Sheeran, P. (2000). Motivational and volitional processes in action initiation: A field study of the role of implementation intentions. Journal of Applied Social Psychology, 30, 780797.

Orbell, S., Hodgkins, S. \& Sheeran, P. (1997). Implementation intentions and the theory of planned behavior. Personality and Social Psychology Bulletin, 23, 945-954.

Powers, T. A., Koestner, R. \& Topciu, R. A. (2005). Implementation intentions, perfectionism, and goal progress: Perhaps the road to hell is paved with good intentions. Personality and Social Psychology Bulletin, 31, 902-912.

Rock, D. \& Schwartz, J. (2006). The Neuroscience of Leadership. In: Strategy and Business, 05.2006.

Schaal, B. \& Gollwitzer, P. M. (2000). Planen und Zielverwirklichung: Die Bedeutung antizipierter Zukunt beim Widerstehen befürchteter Versuchungen. In J. Moeller \& B. Strauss (Eds.), Psychologie der Zukunft (pp. 149-170). Göttingen: Hogrefe.

Schweiger-Gallo, I., Keil, A., McCulloch, K. C., Rockstroh, B. \& Gollwitzer, P. M. (2009). Strategic automation of emotion regulation. Journal of Personality and Social Psychology, 96, 11-31. 
Sheeran, P. \& Orbell, S. (1999). Implementation intentions and repeated behaviour: Augmenting the predictive validity of the theory of planned behaviour. European Journal of Social Psychology, 29, 349-369.

Sheeran, P., Webb, T. L. \& Gollwitzer, P. M. (2005). The interplay between goal intentions and implementation intentions. Personality and Social Psychology Bulletin, 31, 87-98.

Stadler, G., Oettingen, G. \& Gollwitzer, P. M. (2009). Physical activity in women. Effects of a selfregulation intervention. American Journal of Preventive Medicine, 36, 29-34.

Steller, B. (1992). Vorsätze und die Wahrnehmung günstiger Gelegenheiten. München: tuduv-Verlag.

Stewart, B. D., \& Payne, K. B. (2008). Bringing automatic stereotyping under control: Implementation intentions as efficient means of thought control. Personality and Social Psychology Bulletin, 34, 1332-1345.

Stroop, John Ridley (1935). Studies of interference in serial verbal reactions. Journal of Experimental Psychology 18: 643-662.

Trötschel, R. \& Gollwitzer, P. M. (2007). Implementation intentions and the willful pursuit of prosocial goals in negotiations. Journal of Experimental Social Psychology, 43, 579-598.

Webb, T. L. \& Sheeran, P. (2003). Can implementation intentions help to overcome ego-depletion? Journal of Experimental Social Psychology, 39, 279-286.

Webb, T. L. \& Sheeran, P. (2004). Identifying good opportunities to act: Implementation intentions and cue discrimination. European Journal of Social Psychology, 34, 407-419.

Webb, T. L. \& Sheeran, P. (2006). Does changing behavioral intentions engender behavior change? A meta-analysis of the experimental evidence. Psychological Bulletin, 132, 249-268

Webb, T. L. \& Sheeran, P. (2008). Mechanisms of implementation intention effects: The role of goal intentions, self-efficacy, and accessibility of plan components. British Journal of Social Psychology, 47, 373-395.

Webb, T. L., Christian, J. \& Armitage, C. J. (2007). Helping students turn up for class: Does personality moderate the effectiveness of an implementation intention intervention? Learning and Individual Differences, 17, 316-327. 